\title{
How to Write a Protocol: Part 1
}

\author{
Kathy Thomas, CNMT, FSNMMI-TS, and Mary Beth Farrell CNMT, FSNMMI-TS
}

CE credit: For CE credit, you can access the test for this article, as well as additional JNMT CE tests, online at https://www.snmmilearningcenter.org. Complete the test online no later than March 2018. Your online test will be scored immediately. You may make 3 attempts to pass the test and must answer $80 \%$ of the questions correctly to receive $1.0 \mathrm{CEH}$ (Continuing Education Hour) credit. SNMMI members will have their CEH credit added to their VOICE transcript automatically; nonmembers will be able to print out a CE certificate upon successfully completing the test. The online test is free to SNMMI members; nonmembers must pay $\$ 15.00$ by credit card when logging onto the website to take the test.

Clinical imaging protocols play an important role in the provision of high-quality care in nuclear medicine. It is imperative that all nuclear medicine facilities have protocols for every procedure performed. However, creating protocols that are detailed, unambiguous, and consistent is often easier said than done. Properly written protocols help to ensure that nuclear medicine procedures are performed in a standardized, reproducible manner so that patients receive high-quality care. This 2-part article provides technologists with a framework for composing comprehensive protocols. Part 1 discusses the secrets to successfully composing protocols ensuring they are detailed and step-by-step along with the importance of basing protocols on evidence from published guidelines and peer-reviewed literature. The components and important aspects of clinical imaging protocols are detailed.

Key Words: protocol; evidence-based; accreditation; guidelines; standardization

J Nucl Med Technol 2015; 43:1-7

DOI: 10.2967/jnmt.114.147793

C linical imaging protocols play an important role in the provision of high-quality care in nuclear medicine. It is imperative that all nuclear medicine facilities have sitespecific protocols for every procedure performed. However, creating protocols that are detailed, unambiguous, and consistent is often easier said than done. Adding to the difficulty of creating protocols is the fact that education on writing protocols is not included in the curriculum of most nuclear medicine programs and that, in general, nuclear medicine technologists are more often experts in their technical skills than in literary skills. The goal of this article is to provide technologists with a framework for composing comprehensive, site-specific protocols. Included in part 1 is

\footnotetext{
Received Aug. 26, 2014; revision accepted Nov. 19, 2014.

For correspondence or reprints contact: Kathy S. Thomas, CNMT, FSNMMI-TS, 22507 NE 167th Ave., Battle Ground, WA 98604.

E-mail: ksthomas0412@msn.com

Published online Jan. 22, 2015.

COPYRIGHT (C 2015 by the Society of Nuclear Medicine and Molecular Imaging, Inc.
}

a discussion of the secrets to successfully composing protocols to ensure they are detailed and step-by-step, along with a discussion of the importance of basing protocols on evidence from published guidelines and peer-reviewed literature. The components and important aspects of clinical imaging protocols are detailed. Part 2 will cover protocols for exercise and pharmacologic stress testing, therapy, and quality control.

A protocol is defined as a detailed plan for a medical experiment, treatment, or procedure. The goal of any protocol is to provide detailed structure for how to manage the patient and how to perform the procedure. It should provide precise instructions on what should be done, how, when, to whom, and why. Protocols define workflow, create uniformity, help to eradicate omissions, and reduce confusion. When a procedure is performed in a standardized, reproducible manner, inter- and intraoperator variability is reduced, ensuring that each patient study is of optimal quality and every patient receives the same quality of service.

The importance of standardization can be clearly illustrated using PET scanning. The standardized uptake value, used to differentiate benign from malignant tumors and assess response to therapy, requires precision and reproducibility. Precision begins with accurate data, including the patient's height and weight (measured on the day of imaging), dose activity, time of measurement, and time of injection. Standardized uptake values will change over time (1). Comparable quantification of standardized uptake values at different time points relies on the production of reproducible data from one time point to the next. A standardized protocol defines the steps to acquire precise, reproducible data to support optimal patient care.

Protocols are also important for other reasons. They provide an outline for technologist training on performing procedures along with a mechanism to assess competency in performing procedures. A protocol provides a written record of the expected care to be provided to a patient. As long as the protocol adheres to best patient practices and complies with all federal, state, and local laws and regulations, it may provide a measure of protection in medical malpractice negligence claims $(2,3)$. Finally, protocols play an important 
role in accreditation, as the submission and evaluation of clinical protocols is an essential consideration in the accreditation process $(4,5)$.

\section{WHAT MAKES A PROTOCOL GOOD?}

A good protocol thoroughly and specifically describes what is to be done. It should literally be in "cookbook" format to guide the technologist step-by-step through the procedure. When one is composing a protocol, it is helpful to imagine that all the technologists at the facility won the lottery and quit. What would a newly hired technologist need to know to image a patient in the exact same manner as in the past to produce the same results? Consistency in protocol performance is critical, especially if a patient is being imaged for follow-up, when accurate, reproducible results are mandatory.

Protocols must be evidence-based and constructed from well-recognized references (6). Most notably, the published imaging guidelines from the Society of Nuclear Medicine and Molecular Imaging, American Society of Nuclear Cardiology, and American College of Radiology should be used (7-9). In the absence of imaging guidelines, articles from relevant, peer-reviewed published journals should be used. As a case in point, currently there are no published guidelines for myocardial sympathetic innervation imaging with ${ }^{123}$ I-metaiodobenzylguanidine. However, there are several published journal articles on which to base a protocol.

Using the radiopharmaceutical package insert and prescribing information is helpful when creating protocols, along with monographs and educational materials issued by the manufacturer. As an example, ${ }^{123}$ I-ioflupane (DaTscan; GE Healthcare) imaging for Parkinson disease is a relatively new procedure lacking published guidelines. Nevertheless, there is a wealth of imaging information located on the manufacturer's website. (10) Additionally, when the Food and Drug Administration approves new equipment or technologies, oftentimes there may not be an abundance of published literature and the manufacturer's recommendations must be relied on. This was the case when wide-beam reconstruction software was initially available for use.

Finally, it is helpful to consult the regulations from the Nuclear Regulatory Commission and agreement states when developing protocols (11).

\section{THE WHO, WHEN, AND WHAT OF PROTOCOLS}

Who should be responsible for writing a protocol? The answer is any and all stakeholders in a procedure (12). These may include the medical director, interpreting staff, radiation safety officer, physicist, staff technologists, nurses, administration, and perhaps auxiliary personnel such as receptionists or transcriptionists. However, in the end it is the technologist who will most likely be doing the actual writing. Therefore, it is important that the technologist obtain input and buy-in from all stakeholders.
The medical director must approve, sign, and date all protocols. Depending on the practice setting, approval by the radiation safety officer may also be required. Official approval of a protocol is important because it is essentially a standing order allowing the protocol to be performed under the direction of a qualified physician. In the absence of authorized protocols, individual prescriptions and directions from a physician are required for each patient.

This is particularly true with regard to the administration of radiopharmaceuticals. If a facility does not have approved protocols prescribing the identity, amount or dose range, and route of administration of each radiopharmaceutical for each imaging procedure, then there must be a written prescription by an authorized user for each patient (13).

Additionally, protocols should be updated and approved by the medical director annually. This helps to ensure that protocols follow current guidelines or best practices and accurately reflect the procedure as currently performed. Whenever a revision is made to an existing protocol, the revision date should be noted on the revised copy and a copy of the previous protocol archived with a notation citing the dates the protocol was in force, that is, "start date" and "end date." Ideally, archived protocols should be in a protected form that minimizes the possibility of tampering. As noted, a facility's protocol is a written record of expected care for a patient and might be important documentation in a negligence claim (14).

Additionally, it is important to remember that protocols are not limited to imaging procedures. Department protocols not only include all clinical imaging examinations such as bone scans, gastric emptying studies, or myocardial perfusion imaging but also exercise and stress test procedures. There should also be detailed, specific protocols for all therapy procedures. Finally, yet equally important, there should be protocols for quality control tests performed on imaging and nonimaging equipment.

\section{COMPONENTS OF A WELL-WRITTEN PROTOCOL}

The required components for a protocol vary by type of protocol. The necessary components for a clinical imaging protocol are different from the components of a therapy protocol. For example, informed consent or a written directive is not needed when performing diagnostic imaging but is critical for therapy. The required components for clinical imaging protocols are detailed below, whereas protocols for cardiac stress testing, therapy, and quality control will be included in part 2 of this article.

\section{Indications and Contraindications}

Each clinical imaging examination should have specific indications and contraindications stated in a simplified manner. Clinical indications list the possible reasons for the test based on clinical evidence. An indication is the clinical question or the reason for performing the study. Indications should be based on the net benefit to a patient related to the pretest and posttest probability of a disease or 
condition. For instance, a ventilation-perfusion lung scan is indicated for the detection of pulmonary embolism in patients with sudden onset of shortness of breath and chest pain that worsens with deep breath (15).

Contraindications are the specific reasons that a procedure should not be used because it may be harmful to the patient or there may be no benefit. By way of illustration, a gastrointestinal bleed study is contraindicated for a patient not actively bleeding and should not be performed, as a gastrointestinal bleed study shows only active bleeding and cannot detect sites of prior bleeding.

\section{Patient Preparation, Education, and Instructions}

Appropriate patient preparation before an imaging examination is crucial to the accuracy and quality of the study. All protocols should specifically list requirements for food or diet restrictions and specific timelines for any restrictions noted. If the patient is not allowed to eat or drink, it should be noted whether water is allowed. On the other hand, there are some tests, such as a renogram, for which proper patient hydration before the scan is important. Instructions for hydration should be specifically detailed in the protocol. For example, in the case of a renogram, the protocol should cite, "hydrate the patient $1 \mathrm{~h}$ before the examination with 300-500 mL (10$16 \mathrm{oz}$ ) of water over $30 \mathrm{~min} . "$

Protocols should specifically state whether certain foods must be avoided before the test and the specific period for abstinence. It is well recognized that caffeine and caffeinecontaining products should be avoided 12-18 h before vasodilator pharmaceutical stress. Caffeine interferes with adenosine binding sites, preventing the intracellular uptake of adenosine and precluding blood vessel dilation. If a patient consumes caffeine before undergoing vasodilator stress, there is a risk for a false-negative myocardial perfusion imaging result.

The withholding or nonwithholding of medications can be vital to the results of a test. For example, thyroid uptake values may be affected in patients taking thyroid hormones and antithyroid medications. A typical protocol recommends withholding antithyroid drugs for 2-4 d, T4 therapy for 4-6 wk, and T3 therapy for $2 \mathrm{wk}$. Also, it is important for the protocol to not only state the types of medications to be avoided but also list specific common examples. For instance, a gastric emptying protocol that simply states to withhold prokinetic agents is unhelpful if the technologist is unaware of which medications are considered prokinetic, such as metoclopramide (Reglan; Schwarz Pharma), and thus cannot provide proper patient instructions before the test.

Protocols should also provide patient instructions for medications administered before a test, such as the administration of potassium iodide before adrenal imaging with ${ }^{131}$ I-metaiodobenzylguanidine.

Other types of patient preparation such as bowel preparation or administration of oral laxatives; skin prep- aration; and wound care, such as changing or removing dressings or casts, should also be stated in the protocol. In particular, before prostate tumor imaging with ${ }^{111} \mathrm{In}$ capromab pendetide (ProstaScint; Cytogen Corporation), it is recommended that bowel-cleansing preparation $24 \mathrm{~h}$ before imaging and an enema the morning of the imaging be performed.

If no patient preparation or restrictions are required, this should also be clearly stated in the protocol.

It is helpful to detail other patient education or instructions in the protocol. This could include instructions to wear comfortable shoes instead of high heels for an exercise stress test or instructions to alert the patient that follow-up scans at 24, 48, or $72 \mathrm{~h}$ after injection are required for ${ }^{131} \mathrm{I}$ for whole-body scanning.

\section{Radiopharmaceutical Identity, Dosage, and Route of Administration}

The administration of a radiopharmaceutical to a patient must be prescribed by an authorized user, either by an individually written prescription or by a standardized protocol. The standardized protocol acts as a standing order and specifically states the prescribed radiopharmaceutical identity, amount or dose range, and route of administration (e.g., oral, inhalation, subcutaneous, intramuscular, intravenous, or intradermal).

It is also recommended that radiation dosimetry such as the effective dose and critical organ dose be included in the protocol for each prescribed radiopharmaceutical.

\section{Adjunct Medications}

All adjunct medications used as part of the imaging procedure must be stated in the protocol. Again, this should include the medication identity, dose, and route of administration along with the timing of administration, patient instructions, requirements for patient monitoring, and precautions for side effects or restrictions. Adjunct medications include all pharmacologic stress agents and medications used to treat side effects, such as aminophylline or esmolol. Adjunct medications also include medications used to enhance an imaging study or produce a physiologic effect, such as cholecystokinin or morphine used during a hepatobiliary study or furosemide or captopril used during renal imaging.

\section{Camera Setup}

The technical parameters for any imaging study are often ignored in protocols but are critically important. Image quality and diagnostic accuracy and reproducibility depend on the use of appropriate image acquisition parameters. Important camera setup parameters that should be listed in a protocol are detailed in Table 1.

\section{Patient Position and Camera Position}

Patients are often imaged in various positions. For example, a study may be performed with the patient in the supine, prone, or right or left lateral decubitus position. Specific patient positioning instructions such as arms up or 


\begin{tabular}{|c|c|}
\hline Parameter & Setup details \\
\hline Collimator & $\begin{array}{l}\text { Collimator-specific properties such as thickness (depth), width (bore), and angulation (diverging/ } \\
\text { converging) affect sensitivity and resolution of acquisition. Collimator selection (e.g., low, medium, } \\
\text { and high energy; low or high resolution; or high sensitivity) depending on isotope used and } \\
\text { administered dose should be stated. Proper collimator selection can enhance quality of images and } \\
\text { data collected, as is so when high-sensitivity collimator is used to increase counting rate during } \\
\text { first-pass study. }\end{array}$ \\
\hline $\begin{array}{l}\text { Energy setting and } \\
\text { window }\end{array}$ & Correct photopeak and energy window setting should be used. \\
\hline Acquisition type & Whether study is static, dynamic, planar, or SPECT should be described. \\
\hline $\begin{array}{l}\text { Orbit, orbit type, and } \\
\text { number of projections }\end{array}$ & $\begin{array}{l}\text { For SPECT studies, protocol should state whether } 180^{\circ} \text { (as is common in cardiac studies) or } 360^{\circ} \text { orbit } \\
\text { is used. Orbit type, such as circular or body contouring, along with number of stops or projections } \\
\text { should also be stated. }\end{array}$ \\
\hline Acquisition time or counts & $\begin{array}{l}\text { Because count density directly affects diagnostic quality, acquisition time or minimum counts should } \\
\text { be specified. There may be instances (e.g., with bone scanning) in which counts are obtained in one } \\
\text { view, and time for that image is used for remaining views. }\end{array}$ \\
\hline $\begin{array}{l}\text { Matrix, pixel size, and } \\
\text { zoom }\end{array}$ & $\begin{array}{l}\text { For most nuclear medicine studies, matrix and pixel size are relatively standard. Dynamic studies are } \\
\text { often acquired using } 64 \times 64 \text { or } 128 \times 128 \text { matrix, whereas static images commonly use } 254 \times 254 \\
\text { matrix. However, for myocardial perfusion imaging, pixel size (smallest discrete unit of image matrix) } \\
\text { and matrix size play direct role in count statistics and resolution. Smaller pixel size required for } \\
\text { large-field-of-view cameras is accomplished by adjusting camera zoom to be able to detect } \\
\text { perfusion defects. }\end{array}$ \\
\hline Gating & $\begin{array}{l}\text { For cardiac gated studies, number of frames per cardiac cycle should be stated. Ejection fraction } \\
\text { calculated from study acquired using } 8 \text { frames per cycle vs. } 16 \text { frames per cycle will result in } \\
\text { different values. R-R acceptance window, which is particularly important in patients with irregular } \\
\text { heart rates, should also be stated. Wide R-R window used for myocardial perfusion study results in } \\
\text { count statistics different from narrow R-R window used for equilibrium radionuclide studies. }\end{array}$ \\
\hline
\end{tabular}

down for SPECT studies, bra on or off for myocardial perfusion studies, or neck extended for thyroid imaging should be stated to ensure the reproducibility and accuracy of a study.

The camera position such as starting angle, detector configuration, and tilt should be included in the protocol. This is the case for the performance of an equilibrium radionuclide study, for which the protocol should include instructions for the use of a mild $10^{\circ}-15^{\circ}$ caudal tilt on the left anterior oblique position to separate the left atrium from the left ventricle. The protocol should state whether the target organ or area of interest should be centered in the field of view or positioned off-center. There are many procedures in which the target organ is positioned off-center or at the edge of the field of view to allow a physiologic process to be monitored such as during hepatobiliary imaging, in which the liver is positioned superiorly in the field of view. Additionally for most procedures, the camera is placed as close to the patient as possible. However, for some procedures, such as thyroid uptake or any procedure using a pinhole collimator, a standard patient-to-detector distance is desirable and this distance must be stated in the protocol.

\section{Acquisition Instructions}

Camera- and computer-specific acquisition instructions must be detailed in the protocol to include views, number of views, and time or counts per view. Specific procedural instructions should be described; for example, for studies such as diuretic renography, pre- and postvoid kidney and bladder images should be clearly described. Additional views that are optional or may help differentiate pathologic findings should be suggested in the protocol, such as the use of oblique views of the pelvis during skeletal imaging to differentiate between structures perpendicular to the field of view. Appropriate anatomic landmarks and the use of anatomic markers should be clearly described in the protocol.

Appropriate injection-to-imaging time is critical to the results of a study and must be specifically noted. Some procedures, such as renography or hepatobiliary imaging, must be started at the time of injection to assess perfusion or a physiologic process. However, for other procedures, injection-to-imaging time is delayed to allow adequate uptake of the tracer and clearance from background, such as the clearance from background in a bone imaging study.

Finally, if support from other departments is needed, such as for intrathecal injection of ${ }^{111}$ In-diethylenetriamine pentaacetic acid for cisternography or intradermal injections of ${ }^{99 m}$ Tc-sulfur colloid for lymphoscintigraphy, steps for coordination between the departments should be described in the protocol.

\section{Processing Instructions}

Protocols should specifically detail any postacquisition manipulation or processing. Because image processing is done for multiple reasons, such as to enhance the image, to reorient or reorganize, to measure specific physiologic 
parameters, or to allow comparison with established normal populations, there should be instructions for how the desired type of processing is accomplished (16). As an example, an initial bone flow study is acquired at 1 frame per second. However, images are often reformatted by adding 3 images to create a dynamic set with improved count density.

Another processing technique commonly performed that should be intricately described is count or image subtraction. Count or background subtraction, in which a numeric value from an area of interest is subtracted from all pixels and images, is performed during the processing of many studies. On the other hand, clinically useful information is obtained from the subtraction of one image from another, as occurs for dual-isotope parathyroid imaging, in which ${ }^{99 \mathrm{~m}}$ Tc-pertechnetate is subtracted from ${ }^{99 \mathrm{~m}} \mathrm{Tc}$-sestamibi.

The steps involved in any image quantitation should be clearly spelled out. The exact placement, size, and shape of any regions of interest must be delineated. For example, when a region of interest is being drawn over the kidney, should the area include the whole kidney and renal pelvis, the cortex and collecting system, or simply the cortex? The protocol should also carefully detail the placement of background regions of interest, as different results are obtained due to variances in region-of-interest placement. This issue can be clearly demonstrated by moving the background region for an equilibrium radionuclide study into an area of higher count density, resulting in a falsely elevated ejection fraction (Fig. 1). Protocol steps for the creation of any time-activity curves and calculation of quantitative values should be clearly explained as well for procedures such as the calculation of gallbladder ejection fraction computed from a hepatobiliary study.

For SPECT studies, protocols should describe the type of reconstruction-that is, whether filtered-backprojection or iterative reconstruction is used. Other reconstruction parameters such as type of filter and cutoff value, image reorientation, and display (e.g., transaxial/longitudinal/oblique images for general nuclear medicine verses short axis/horizontal long axis/vertical long axis for myocardial perfusion imaging) should be explained. Protocols should also describe any steps and procedures for attenuation correction or motion correction that may be performed.

Additional processing details that should be included in the protocol include camera- and computer-specific instructions on image and data display. The protocol should contain instructions for how images should be labeled (e.g., patient name, date of birth, patient identifier, date of study, time interval, laterality, and anatomic markers). There should also be instructions for how the images are presented for physician interpretation, such as from hard copy or uploaded to a PACS.

\section{PRECAUTIONS}

The final component of a protocol should include a discussion of any special precautions that should be

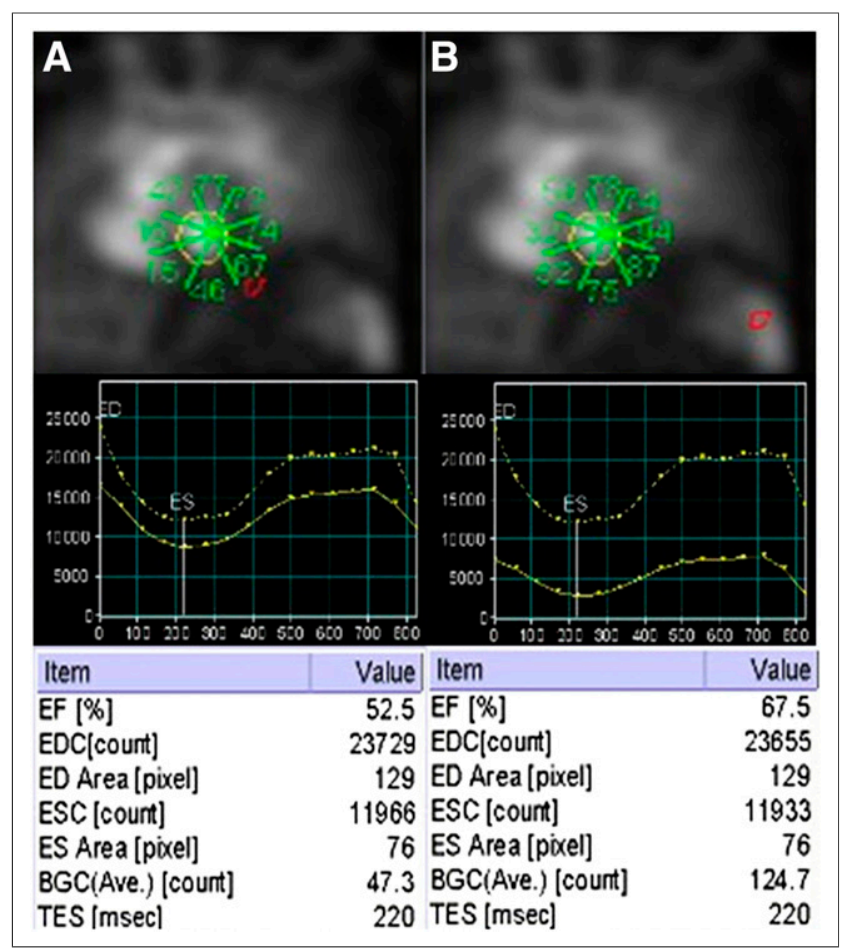

FIGURE 1. Demonstration of importance of protocol defining precise placement of region of interest. (A) Background region of interest is correctly placed below myocardium in area of low counts, resulting in ejection fraction of $52.5 \%$. (B) Background region is incorrectly placed in area of high counts on spleen, resulting in ejection fraction of $67.5 \%$. BGC = background count; ED = enddiastole; EDC = end-diastolic count; EF = ejection fraction; ES = end-systole; ESC = end-systolic count; TES = time to end-systole.

taken during or after the procedure. For example, when ${ }^{99 \mathrm{~m}}$ Tc-macroaggregated albumin is injected for a perfusion lung scan, blood should not be pulled back into the syringe because clotting may occur, resulting in hot spots on the images. As another example, if sedation is used during ${ }^{18}$ F-FDG PET imaging, the patient should be monitored and not allowed to drive.

\section{SUMMARY}

This article details the recommended components of a well-written clinical protocol; however, for most nuclear medicine technologists, the ability to apply these recommendations may seem somewhat overwhelming at first glance. For those who find the "show me" educational process somewhat easier to follow, the bone scan protocol in Figure 2 is an example of a successfully written protocol.

\section{CONCLUSION}

Protocols should not be viewed as inflexible requirements or absolute standards of care. Every patient is different and presents with a unique clinical scenario and individual needs. Thus, a scanning procedure must be 


\section{Indications}

1. Diagnosis of the following:

a. Neoplastic disease

b. Occult fracture

c. Stress reaction/stress fracture

d. Avascular necrosis

e. Arthritis

f. Reflex sympathetic dystrophy

g. Bone infarcts

h. Bone graft viability

i. Paget disease

j. Unexplained bone pain

2. Evaluation of distribution of osteoblastic activity before radionuclide therapy for bone pain

Note: No appropriateness criteria have been developed to date for ${ }^{18} \mathrm{~F}$ bone scans; however, the indications listed may be appropriate in certain individuals.

\section{Contraindications}

1. Pregnant/breast-feeding: Pregnancy must be excluded in accordance with local institutional policy. If the patient is breast-feeding, appropriate radiation safety instructions should be provided.

2. Use of oral contrast (barium) within 24 to 48 hours of the procedure (per the interpreting physician's preference).

Patient preparation/education

1. The patient may eat and take medications as necessary before the procedure.

2. Obtain a focused history to include the following:

a. Clinical indication for the study, including current symptoms

b. History of fractures, trauma, and associated bone abnormalities or pain

c. History of current or previous therapeutic protocols that may affect the bone scan

d. History of surgical procedures that may affect the bone scan

e. Review of current medications that may affect distribution of the tracer

f. Confirmation of pregnancy status and/or lactation

Radiopharmaceutical identity, dose, and route of administration

\begin{tabular}{|c|c|c|}
\hline Identity & Dose & $\begin{array}{c}\text { Route of } \\
\text { administration }\end{array}$ \\
\hline $\begin{array}{l}{ }^{18} \mathrm{~F} \text {-sodium } \\
\text { fluoride }\end{array}$ & $\begin{array}{l}185 \mathrm{MBq}(5 \mathrm{mCi}) \\
\text { Range: } 185-370 \mathrm{MBq}(5-10 \\
\mathrm{mCi})\end{array}$ & Intravenous \\
\hline $\begin{array}{l}\text { Pediatric dose: } \\
{ }^{18} \mathrm{~F} \text {-sodium } \\
\text { fluoride }\end{array}$ & $\begin{array}{l}2.2 \mathrm{MBq} / \mathrm{kg}(0.06 \mathrm{mCi} / \mathrm{kg}) \\
\text { Minimum administered } \\
\text { activity: } 18.5 \mathrm{MBq}(0.5 \mathrm{mCi})\end{array}$ & Intravenous \\
\hline
\end{tabular}

Pharmaceutical identity, dose, and route of administration

None

\begin{tabular}{|c|c|c|}
\hline & & $\begin{array}{l}\text { Standard/ } \\
\text { preferred/ } \\
\text { optional }\end{array}$ \\
\hline Camera type & PET or PET/CT & Standard \\
\hline Energy peak & $511 \mathrm{keV}$ & Standard \\
\hline $\begin{array}{l}\text { Injection to } \\
\text { imaging time }\end{array}$ & 90-120 minutes & Standard \\
\hline $\begin{array}{l}\text { Attenuation } \\
\text { correction }\end{array}$ & $\begin{array}{l}\text { PET: cesium or } \\
\text { germanium sources } \\
\text { PET/CT: CT acquisition }\end{array}$ & Standard \\
\hline Patient position & Supine & Standard \\
\hline Arm position & Indication specific & Standard \\
\hline Acquisition mode & $\begin{array}{l}\text { 2-dimensional or 3- } \\
\text { dimensional }\end{array}$ & Standard \\
\hline Bed positions & $\begin{array}{l}\text { Adequate to cover whole } \\
\text { body or specific area of } \\
\text { interest }\end{array}$ & Standard \\
\hline Time/bed position & $\begin{array}{l}\text { 2-dimensional: 2-5 } \\
\text { minutes } \\
\text { 3-dimensional: } 2-3 \\
\text { minutes }\end{array}$ & $\begin{array}{l}\text { Standard } \\
\text { Standard }\end{array}$ \\
\hline View & $\begin{array}{l}\text { Top of the head to the } \\
\text { toes }\end{array}$ & Standard \\
\hline
\end{tabular}

\section{Acquisition parameters: $\mathrm{PET} / \mathrm{CT}$}

Refer to the manufacturer's recommendations for CT acquisition parameters.

\section{Acquisition instructions: PET}

1. Acquire whole body images and images for limited areas of interest by placing the patient in the supine position on the scanning table.

2. To avoid bladder artifact, have the patient void before imaging and between image sets.

3. Arm position is dependent on clinical indications. The arms may be at the patient's side for whole body imaging or elevated when the scan is limited to the axial skeleton.

\section{Processing instructions}

1. Appropriate reconstruction parameters will depend on the acquisition mode (2-dimensional or 3-dimensional).

2. Iterative reconstruction is most often used for clinical applications in either acquisition mode.

3. Refer to the manufacturer's guidelines for reconstruction protocols for emission data that correct for detector efficiency (normalization), system dead time, random coincidences, scatter attenuation, and sampling nonuniformity.

4. Appropriately scale data and display in transaxial, coronal, and sagittal planes as well as a rotating maximum intensity projection image.

\section{Precautions}

\section{None}

FIGURE 2. ${ }^{18} \mathrm{~F}$ bone scintigraphy protocol demonstrating all components of clinical protocol. 
modified to accommodate the patient and to ensure that the results of the examination answer the clinical question posed by the referring physician. For instance, the protocol may call for the patient to lie supine on the imaging table. However, if the patient is severely claustrophobic, adequate results may be obtained by placing the camera under the patient and imaging in the prone position. The ultimate judgment regarding the use of protocols rests with the nuclear medicine technologist working under the direction of the authorized user or interpreting physician. Let there be no doubt that writing a protocol is challenging both intellectually and technically. It requires thorough investigation and research along with meticulous attention to detail. There may be times when "borrowing" a protocol from another experienced facility may be advantageous. This may be the one time that plagiarizing is acceptable, as long as the protocol is customized to reflect exact procedures as performed in the facility where it will be applied.

This article has provided technologists with a framework for composing appropriate protocols. The secret to successfully creating protocols is to make sure they are detailed, step-bystep, and written in simple language in a manner similar to a cookbook. Protocols should be site- and equipment-specific. It is important that protocols be evidence-based following the best practice recommendations of published guidelines and contain all necessary components. Properly written protocols help to ensure that nuclear medicine procedures are performed in a standardized, reproducible manner so that patients receive high-quality care. Part 2 of this article will address writing protocols for cardiac stress testing, therapy, and quality control.

\section{REFERENCES}

1. Christian PE, Waterstram-Rich K. Nuclear Medicine and PET/CT Technology and Techniques. Philadelphia, PA: Mosby Elsevier; 2007:336.

2. Nuclear medicine technologist scope of practice and performance standards. Society of Nuclear Medicine and Molecular Imaging website. http://snmmi. files.cms-plus.com/NMT_Scope_of_Practice_Clinical_Performance_Standards_ FINAL_6-2013.pdf. Accessed January 20, 2015.

3. The practice standards for medical imaging and radiation therapy. American Society of Radiologic Technologists website. http://media.asrt.org/pdf/ governance/practicestandards/ps_nm.pdf. Accessed January 9, 2015.

4. Intersocietal Accreditation Commission Policies and Procedures. Ellicott City, MD: Intersocietal Accreditation Commission; 2012.

5. Nuclear Medicine Accreditation Program Requirements. Reston, VA: American College of Radiology; 2014:13.

6. Eisenbert R. Radiology and the Law. New York, NY: Springer; 2003.

7. Procedure standards. Society of Nuclear Medicine and Molecular Imaging website. http://www.snmmi.org/ClinicalPractice/content.aspx ?ItemNumber $=6414 \&$ navItemNumber $=10790$. Accessed January 9, 2015.

8. Clinical guidelines and quality standards. American Society of Nuclear Cardiology website. http://www.asnc.org/content_184.cfm?navID=73. Accessed January 9, 2015.

9. Practice parameters and technical standards. American College of Radiology website. http://www.acr.org/Quality-Safety/Standards-Guidelines. Accessed January 9, 2015.

10. DaTscan ${ }^{\mathrm{TM}}$ ioflupane I123 injection. GE Healthcare website. http://us.datscan. com/imagers/. Accessed January 9, 2015.

11. Part 35-medical use of byproduct material. U.S. Nuclear Regulatory Commission website. http://www.nrc.gov/reading-rm/doc-collections/cfr/part035/. Accessed January 9, 2015.

12. Owen A, Hogg P, Nightingale J. A critical analysis of a locally agreed protocol for clinical practice. Radiography. 2004;10:139-144.

13. Siegel J. Guide for Diagnostic Nuclear Medicine and Radiopharmaceutical Therapy. Reston, VA: Society of Nuclear Medicine; 2004.

14. Berlin L. Malpractice issues in radiology: standards, guidelines, and roses. AJR. 2003; 181:945-950.

15. Farrell M, Mantel E, Basso D, Thomas K, Kerr B. Quick Reference Protocol Manual for Nuclear Medicine Technologists. Reston, VA: Society of Nuclear Medicine and Molecular Imaging; 2014.

16. Alessi A. Basic myocardial processing techniques. In: Alessi A, Farrell M, Grabher B, et al., eds. Nuclear Cardiology Technology Study Guide. Reston, VA: Society of Nuclear Medicine; 2010:75-77. 